\title{
DARK MATTER ON THE SMALLEST SCALES
}

\author{
E. R. Siegel ${ }^{1}$, G. D’Amico ${ }^{2}$, E. Di Napoli ${ }^{3}$, L. Fu ${ }^{4}$, M. P. \\ Hertzberg $^{5}$, N. T. T. Huong ${ }^{6}$, F. Palorini ${ }^{7}$, A. Sellerholm ${ }^{8}$ \\ ${ }^{1}$ Department of Physics, University of Wisconsin, 1150 University Avenue, Madison, WI 53706, USA \\ ${ }^{2}$ Department of Physics, Scuola Normale Superiore, Pisa 56100, Italy \\ ${ }^{3}$ Department of Physics and Astronomy, University of North Carolina, CB\#3255 Phillips Hall, \\ Chapel Hill, NC 27599, USA \\ ${ }^{4}$ Joint Center of Astrophysics, Shanghai Normal University, 200234 Shanghai, China \\ ${ }^{5}$ Center for Theoretical Physics, MIT, Cambridge, MA 02139, USA \\ ${ }^{6}$ Centre for High Energy Physics, Vietnam National University - Hanoi, 334 Nguyen Trai Street, \\ Hanoi, Vietnam \\ ${ }^{7}$ Universitè C. Bernard, 69662 Villeurbanne cedex, France \\ ${ }^{8}$ Department of Physics, Stockholm University, 10691 Stockholm, Sweden
}




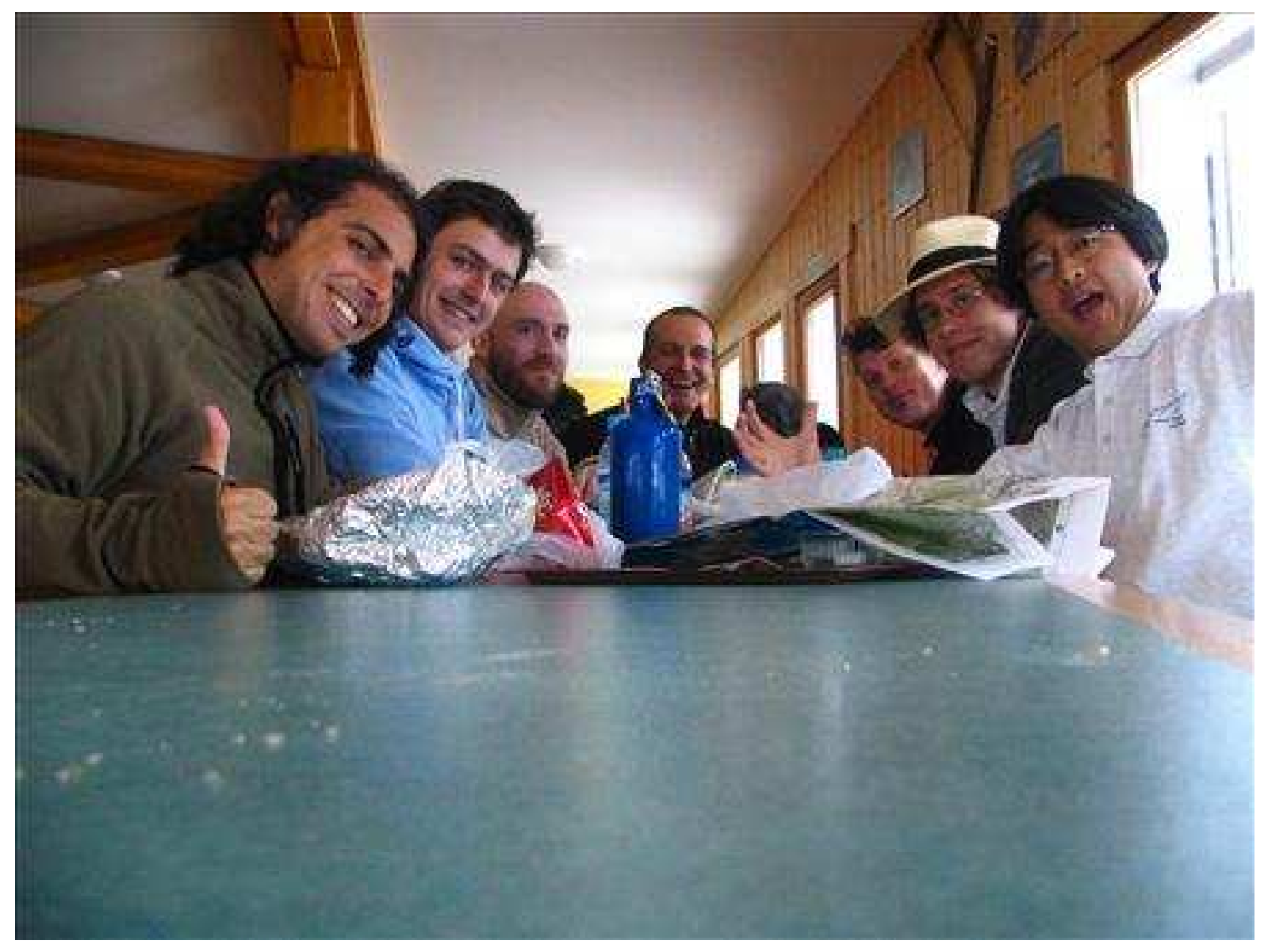




\section{Contents}

1. Introduction

2. The Dark Matter Problem

3. Dark Matter Candidates

4. Small Scale Structure

5. Detectability and Future Work

References 



\begin{abstract}
This work investigates the dark matters structures that form on the smallest cosmological scales. We find that the types and abundances of structures which form at approximately Earth-mass scales are very sensitive to the nature of dark matter. We explore various candidates for dark matter and determine the corresponding properties of small-scale structure. In particular, we discuss possibilities for indirect detection of dark matter through small-scale structure, and comment on the potential of these methods for discriminating between dark matter candidates.
\end{abstract}

\title{
1. Introduction
}

Perhaps the most fundamental goal of cosmological physics is to determine the composition and evolution of the universe. Cosmology has recently reached a state where the vast majority of measurements point towards the same result; the universe is composed of $\sim 5$ per cent baryons, $\sim 20$ per cent dark matter, and $\sim 75$ per cent dark energy. This result is quite puzzling, as dark matter and dark energy have only been detected by their gravitational effects on cosmological scales. While much is known about baryonic structure as well as the particle properties of baryons, much less is known about dark matter structure and its particle properties, and even less is known about dark energy. As a result, the nature of these two latter components of the universe, which compose 95 per cent of its total energy density, are presently the greatest unsolved puzzles in cosmology.

This work investigates the nature of dark matter by examining its effects on cosmologically small scales. In particular, constraining the properties of smallscale structure will constrain the nature of dark matter. The layout of this paper is as follows: section 2 presents an overview of the dark matter problem, focusing on astrophysical evidence. Section 3 identifies the leading particle candidates for dark matter, with a view towards their distinguishing properties. Section 4 describes and contrasts the properties of small scale structure which forms in various dark matter models. Finally, section 5 provides a discussion on possible observable signals which could point towards the nature of dark matter.

\section{The Dark Matter Problem}

The discrepancy between the amount of mass inferred from observations of light and the amount of mass inferred from gravitation on cosmological scales is known 


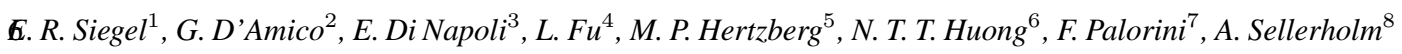

as the dark matter problem. In our solar system, nearly all of the mass is contained in the sun, and so it seems reasonable to assume that light traces mass. Since properties of stars are well known, it is possible to infer the amount of mass present in luminous sources $\left(M_{\star}\right)$, such as stars. From measurements of $M_{\star}$, it is possible to infer the fractional energy density in stars, $\Omega_{\star}$, where $\Omega_{i}$ is defined as the energy density in species $i$ over the critical energy density, $\rho_{c}$. Observations indicate that $\Omega_{\star} \simeq 0.005$ [1], or that luminous matter composes about 0.5 per cent of the total energy density in the universe.

On the other hand, it is also possible to reconstruct the matter density of the universe from its gravitational mass (yielding $\Omega_{m}$ ) instead of its luminous mass $\left(\Omega_{\star}\right)$. Many methods exist for determining $\Omega_{m}$, including extrapolating the matter density from peculiar velocities within galaxy clusters [2], rotational and virial properties of individual galaxies [3], gravitational lensing [4], and from large scale structure [5]. All of these methods yield a range that $0.15<\Omega_{m}<0.35$, consistent with one another at the $2-\sigma$ level. The best measurement of matter density comes from a combination of the latest microwave background data [6] with type Ia supernova data [7], yielding $\Omega_{m}=0.26 \pm 0.02$. This discrepancy between $\Omega_{m}$ and $\Omega_{\star}$ is the dark matter problem; the amount of mass in stars is only 2 per cent of the total matter density! The remaining 98 per cent, the non-luminous matter, is known as dark matter.

\section{Dark Matter Candidates}

Once the existence of dark matter has been established, it becomes a fundamental question to inquire what its nature is. Many candidates have been proposed (see [8] for a review), but a confirmed experimental detection has been thus far elusive. This section therefore investigates the properties and viability of four generic models of dark matter: baryons, neutrinos, thermal relics, and nonthermal relics.

Baryons are the most obvious candidate for dark matter, as baryonic dark matter is observed in the form of planets, low luminosity stars, and diffuse gas clouds. One consequence is that baryonic dark matter will fragment and collapse to form MAssive Compact Halo Objects (MACHOs). Unfortunately, the quantitative effect of Silk damping in large-scale structure [9], the insufficient number of MACHOs [10], and big bang nucleosynthesis [11] all indicate that $\Omega_{m} \simeq 0.04$. Finally, direct observations of X-rays from interacting galaxies show that dark matter does not interact in the same fashion as baryons [12], which means that although baryons compose a significant fraction (about 15 per cent) of the total matter, they cannot be responsible for all of the dark matter.

It is therefore unavoidable that the majority of dark matter be non-baryonic. The standard model contains a candidate for non-baryonic dark matter: the neutrino. However, neutrinos are produced thermally and have very low masses, conflicting with constraints from the Lyman- $\alpha$ forest that limit the mass of thermal dark matter to be at least $2 \mathrm{keV}[13]$. These observations, combined with the constraints on 
neutrino masses, rule out standard model neutrinos as a significant component of dark matter.

Heavier thermal relics are allowed experimentally, however, and particle physics beyond the standard model provides us with many well-motivated dark matter candidates of this type, including the lightest supersymmetric particle and the lightest Kaluza-Klein particle. These particles do not interact either strongly or electromagnetically, and are therefore classified as Weakly Interacting Massive Particles (WIMPs). Thermal relics are defined by the fact that, at some point in the past history of the universe, the particles were in thermal equilibrium with the primordial plasma. At some point, their abundance freezes out (a process known as chemical decoupling), and the number of dark matter particles remains constant thereafter. At a later point, the dark matter particles cease to scatter off of the plasma (kinetic decoupling), and thereafter evolve solely gravitationally.

Nonthermal relics, on the other hand, were never in thermal equilibrium with the primordial plasma. Instead, these dark matter candidate particles can be produced during phase transitions in the early universe. Candidates include axions [14] and massive gravitons [15]. Unlike thermal relics, nonthermal relics evolve only gravitationally from the moment of their creation, receiving only a gravitational imprint from the primordial plasma. As will be discussed in sections 4 and 5 present-day gravitational effects of dark matter have the potential to shed light on its nature, and may be able to distinguish between thermal and nonthermal relics.

\section{Small Scale Structure}

Cosmological structure formation is the key process that allows an understanding of the types and abundances of structures which will form in the universe given a set of initial conditions. While large-scale structure is well understood, small-scale structure is more of a challenge due to the uncertainty of the nature of dark matter and the problems associated with gravitational collapse in the deeply nonlinear regime. The remainder of the work in this paper represents the initial stages of an ambitious research project to probe the nature of dark matter via astrophysical observations on small (i.e., sub-galactic) scales.

Just as baryonic structures are suppressed on mass scales below $\sim 10^{6}$ solar masses $\left(M_{\odot}\right)$ due to Silk damping prior to decoupling, WIMPy structures will be suppressed on scales smaller than the horizon at the time of kinetic decoupling. By contrast, nonthermal relic dark matter will have no such suppression, owing to the fact that they were always decoupled from the primeval plasma. The fluctuations at the time of decoupling are frozen in, and the nonlinear structures on small scales which eventually form from the collapse of dark matter will be very sensitive to the epoch of kinetic decoupling. Hence, the exact mass and abundance of these cosmic microstructures depend very sensitively on the dark matter particle properties. In particular, they are sensitive to dark matter mass, which is directly tied to the times 
of kinetic and chemical decoupling, and to whether the dark matter was produced thermally or nonthermally.

One challenge of understanding small scale structure is to understand the dark matter's evolution through kinetic decoupling. This difficulty is evidenced by the varied results obtained by authors using different approximations [16 17]. We assume that the dark matter is composed of neutralino WIMPs, and follow the approach given by E. Bertschinger [18]. By calculating the transfer functions for cold dark matter fluctuations beginning with the full Boltzmann equations describing scattering between WIMPs and the plasma, the uncertainty created by the aforementioned approximations is eliminated. We begin by treating the dark matter particles as an unperturbed fluid. Analysis allows us to obtain an expression for the temperature at which kinetic decoupling occurs,

$$
T_{d}=0.2528 g_{\mathrm{eff}}^{1 / 8}\left(\frac{m_{\tilde{L}}^{2}-m_{\chi}^{2}}{G_{F} m_{W}^{2} m_{\chi}^{2} \tan \theta_{W}}\right)^{1 / 2}\left(\frac{m_{\chi}^{5}}{m_{\mathrm{Pl}}}\right)^{1 / 4},
$$

where $g_{\text {eff }}$ is the number of thermal degrees of freedom, $G_{F}$ is the Fermi constant, $\theta_{W}$ is the Weinberg angle, and $m_{\chi}, m_{\tilde{L}}$, and $m_{W}$ are the masses of the neutralino, slepton, and $W$ boson. There is an associated time for kinetic decoupling, $t_{d}$. For a slepton mass of $200 \mathrm{GeV}$, this yields a temperature for kinetic decoupling of

$$
T_{d} \simeq 15.7\left(\frac{m_{\chi}}{100 \mathrm{GeV}}\right)^{5 / 4} \mathrm{MeV} \text {. }
$$

By including perturbations in the gravitational field, one can derive the density transfer function for cold dark matter through the epoch of kinetic decoupling. We find that, on scales outside the horizon at kinetic decoupling $\left(k / a<1 / t_{d}\right)$, acoustic oscillations average out, resulting in a logarithmic growth of cold dark matter fluctuations. However, on scales inside the horizon $\left(k / a>1 / t_{d}\right)$, density fluctuations exhibit damped acoustic oscillations, which suppress the formation of structure.

The density transfer function can then be evolved through electron-positron pair annihilation and matter-radiation equality, yielding predictions for small scale cold dark matter structure. The Press-Schechter mass fraction can be derived, and indicates a suppression in the mass of collapsed structure per mass interval on mass scales below $\approx 2.3 M_{d}$, where $M_{d}$ is the mass contained in a typical density fluctuation at kinetic decoupling. For a neutralino of mass $m_{\chi}=100 \mathrm{GeV}$, this indicates that the number of cold dark matter structures which form on mass scales below approximately 20 Earth masses will be suppressed. The root-mean-squared mass density perturbation containing a mass $M, \sigma(M)$, is suppressed as

$$
\frac{d \sigma(M)}{d \ln M} \propto\left(\frac{M}{M_{d}}\right)^{2 / 3}
$$

for masses $M \ll M_{d}$, which then results in a suppression of WIMP microhalos of equivalent mass when converted into the nonlinear regime. By comparison, nonthermal dark matter such as axions does not have a suppression in its PressSchechter mass fraction, as it was always kinetically decoupled [19]. For axion-like 
dark matter, this would indicate a comparatively much larger number of collapsed structures below about 20 Earth masses, resulting in nonlinear structures we term Nonthermal Axionic Collapsed HalOs (NACHOs).

\section{Detectability and Future Work}

It is unknown whether these dark matter microhalos, regardless of whether they are WIMPs or NACHOs, will be able to survive hierarchical mergers and galactic infall, and thus be found intact within our own galaxy [20]. Recent work [21], however, indicates that this is a quantitative question and not a qualitative one, as tidal stripping is not sufficient to completely destroy all of these collapsed structures. One extremely important question to ask is, "what does small scale structure look like today?" $N$-body simulations yield varied results, and there is no consensus as to the density profiles and core concentrations of these objects. Additionally, the Press-Schechter approach may be invalid, as monolithic collapse may be more important than hierarchical mergers on small scales. Ideally, therefore, detection methods which probe the density profiles and abundances of these microhalos will be able to not only constrain the nature of dark matter, but will provide information about the types of structures which form through the deeply nonlinear regime.

If these microhalos survive intact to the present day, a large number should be present within our own galaxy. We therefore seek to uncover methods to detect these microhalos and investigate their properties. For neutralino dark matter, there will be a significant annihilation cross-section, which could result in an observable gamma-ray signal. For a $100 \mathrm{GeV}$ WIMP forming an Earth mass microhalo with an NFW profile, we calculate a gamma-ray flux of $\sim 10^{22}$ photons $\mathrm{sec}^{-1}$. As abundance estimates indicate that the nearest microhalo should be nearer than the nearest star [20], this flux has the potential to outshine even gamma-rays emitted from the galactic center. Gravitational lensing due to a microhalo transit is also a possible effect. While WIMP microhalos are too diffuse, NACHOs may form much more dense structures [19], which may leave observable lensing signals. One very interesting possibility currently being investigated by two of the authors is that a dark matter microhalo transiting across the line-of-sight from an observer to a pulsar could cause a shift in the pulse arrival time due to the gravitational time delay [22]. Finally, interactions between dark matter microhalos and stars or gas clouds may be important. We note that if a dark matter microhalos of a few Earth masses were gravitationally captured by our sun, it would cause an anomalous acceleration towards the sun at significantly large distances.

Cosmological structures on small scales have the potential to shed light on the nature of dark matter, as many of the methods of detecting small-scale dark matter structure are sensitive to its mass and/or method of production. Success of any of the above methods would provide the first definitive confirmation of the presence of dark matter within our own galaxy. Although a tremendous amount of nonlinear processing has occurred since the creation of dark matter microhalos, present 


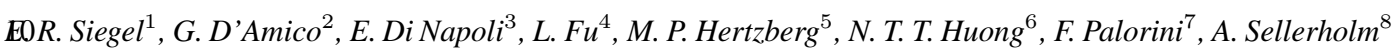

and future searches for small-scale cosmological structures may hold the key to determining the nature of the non-baryonic dark matter in our universe.

We would like to thank the organizers of the 86th Les Houches summer school, Francis Bernardeau and Christophe Grojean, as well as the staff and our colleagues for providing a stimulating and productive working environment. We also acknowledge Sergio Joras, Laura Lopez-Honorez and Gilles Vertongen for their contributions to our group. E.R.S. acknowledges Hai-Ping Cheng and Pierre Ramond for support.

\section{References}

[1] M. Fukugita and P. J. E. Peebles, Astrophys. J. 616, 643 (2004)

[2] R. P. van der Marel, J. Magorrian, R. G. Carlberg, H. K. C. Yee and E. ElLingson, Astron. J. 119, 2038 (2000)

[3] T. G. BRAinerd AND M. A. SPECIAN, Astrophys. J. 593, L7 (2003)

[4] C. Heymans et al., Mon. Not. Roy. Astron. Soc. Lett. 371, L60 (2006)

[5] J. A. PEACOCK et al., Nature 410, 169 (2001)

[6] D. N. SPERGEL et al., arXiv:astro-ph/0603449

[7] A. G. Riess et al., arXiv:astro-ph/0611572

[8] G. Bertone, D. Hooper And J. Silk, Phys. Rept. 405, 279 (2005)

[9] C. J. Miller, R. C. Nichol AND X. L. Chen, Astrophys. J. 579, 483 (2002)

[10] C. Alcock et al. [MACHO Collaboration], Astrophys. J. 499, L9 (1998)

[11] B. D. Fields And K. A. Olive, Nuc. Phys. A 777, 208 (2006)

[12] D. Clowe, M. Bradac, A. H. Gonzalez, M. Markevitch, S. W. Randall, C. JONES AND D. ZARITSKY, arXiv:astro-ph/0608407

[13] M. Viel, J. Lesgourgues, M. G. Haehnelt, S. Matarrese and A. Riotto, Phys. Rev. D 71, 063534 (2005)

[14] R. D. Peccei And H. R. Quinn, Phys. Rev. Lett. 38, 1440 (1977)

[15] S. L. Dubovsky, P. G. Tinyakov and I. I. TKachev, Phys. Rev. Lett. 94, 181102 (2005)

[16] A. M. Green, S. Hofmann And D. J. SchwarZ, JCAP 0508, 003 (2005)

[17] A. Loeb And M. Zaldarriaga, Phys. Rev. D 71, 103520 (2005)

[18] E. Bertschinger, Phys. Rev. D 74, 063509 (2006)

[19] K. M. Zurek, C. J. Hogan AND T. R. QuinN, arXiv:astro-ph/0607341

[20] J. Diemand, B. Moore And J. Stadel, Nature 433, 389 (2005)

[21] T. Goerdt, O. Y. Gnedin, B. Moore, J. Diemand and J. Stadel, arXiv:astro-ph/0608495

[22] E. R. Siegel, J. N. Fry AND M. P. HertZberg, in preparation. 